EOMmun Communication et organisation

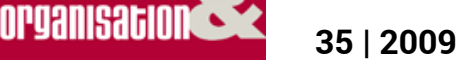

Repenser la communication dans les organisations publiques

\title{
Un laboratoire de recherche en transformation : le LASCO de l'Université Catholique de Louvain
}

Andrea Catellani

\section{OpenEdition}

\section{Journals}

Édition électronique

URL : http://journals.openedition.org/communicationorganisation/868

DOI : 10.4000/communicationorganisation.868

ISSN : $1775-3546$

Éditeur

Presses universitaires de Bordeaux

Édition imprimée

Date de publication : 1 juin 2009

Pagination : 254-263

ISSN : 1168-5549

\section{Référence électronique}

Andrea Catellani, « Un laboratoire de recherche en transformation : le LASCO de I'Université Catholique de Louvain », Communication et organisation [En ligne], 35 | 2009, mis en ligne le 01 décembre 2012, consulté le 15 septembre 2020. URL : http://journals.openedition.org/communicationorganisation/ 868 


\section{Un laboratoire de recherche en transformation : le LASCO de l'Université Catholique de Louvain Andrea Catellani, LASCO, UCL ${ }^{192}$}

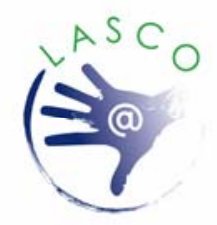

Andrea.Catellani@uclouvain.be

Présenter le LASCO (Laboratoire d'analyse des systèmes de communication des organisations), groupe de recherche sur la communication des organisations de l'université catholique de Louvain (UCL), signifie entrer de plain-pied dans l'histoire de ce domaine d'études en Belgique. Une histoire ancienne, mais ouverte sur le futur et en pleine évolution, que cette brève présentation veut retracer pour permettre de contextualiser l'identité actuelle et les perspectives de ce groupe de recherche ${ }^{193}$.

Les racines : des "techniques de diffusion" à la communication des organisations

Une tradition d'études sur la communication et les relations publiques existe à l'Université catholique de Louvain depuis longtemps. Comme nous avons pu le vérifier sur le site du département de communication ${ }^{194}$ et dans une brochure des années soixante ${ }^{195}$, dès la fin de la Deuxième Guerre (1946) l'université avait introduit des cours sur la presse, organisés dès 1963, en un véritable programme de cours,

\footnotetext{
192 Andrea Catellani est professeur à l'Université Catholique de Louvain.

${ }^{193}$ Une présentation du LASCO a été publiée déjà sur Communication \& Organisation en 2001, peu après la constitution du laboratoire (voir Gryspeerdt 2001). Notre texte veut donc actualiser cette première présentation.

${ }^{194}$ http://www.uclouvain.be/4888.html, consulté le 15 septembre 2009.

195 Nous remercions le Prof. Axel Gryspeerdt, ancien président du LASCO, pour cette information, et le Prof. Pierre de Saint-Georges pour son aide. Nous renvoyons aussi au site du LASCO (http://www.uclouvain.be/206461.html, consulté le 15 septembre 2009) pour de plus amples informations sur l'histoire du laboratoire.
} 
au sein de la licence en sciences politiques et sociales; en 1966 on commence à décerner le titre de licencié en communication sociale. Parmi les autres cours proposés à l'époque, et dédiés en particulier aux différents médias, nous trouvons le cours de Relations publiques, donné par le Prof. Vincent Levaux. Ce cours voulait introduire à la connaissance des politiques menées par «une entreprise, une administration, un groupement ou un homme », pour " entretenir des relations honnêtes et confiantes avec ses employés et ses publics ", en faisant «appel à tous les media de communication sociale et individuelle ${ }^{196}$. Avec le langage et les catégories de l'époque, on présente donc une forme d'enseignement qui couvre un champ comparable à celui des enseignements actuels en communication des organisations (communication interne, externe, stratégique, relations publiques au sens "français » restreint, etc.), selon une perspective orientée vers les exemples nord-américains. L'Université catholique de Louvain a été en effet pionnière en Europe dans ce domaine, en proposant ce cours de Relations publiques à partir de 1958.

A côté de l'enseignement, l'université a commencé très tôt à proposer des activités de recherche. En 1960, au sein de l'Institut des sciences politiques et sociales naît le "Centre des Techniques de Diffusion et des Relations Publiques » (CETEDI), qui peut être considéré comme l'ancêtre du LASCO, en particulier dans sa section "presse et relations publiques ». Ce centre proposait, vers la fin des années soixante, une activité centrée sur l'analyse scientifique des médias et des relations publiques, en s'ouvrant aussi aux études commandées par des entreprises et des organisations extérieures ${ }^{197}$.

En 1975 le «département de communication sociale» devient indépendant, et l'année suivante il déménage de Leuven à Louvain-laNeuve. En 1984 naît le CECOM (Centre d'études de la communication), asbl de l'UCL qui succède au CETEDI dans le champ des «services de formations, de conseils et d'interventions dans le domaine de la communication ", comme le dit son site

\footnotetext{
${ }^{196}$ Brochure, sans date de parution, du Département de Communication et du CETEDI (Centre des techniques de diffusion), Université catholique de Louvain.

${ }^{197}$ L'activité était résumée dans la brochure consultée en quatre points : «mettre au point une méthodologie de la recherche en communication sociale, y compris les relations publiques»; "poursuivre des recherches systématiques et scientifiquement contrôlées, dans les domaines des media et des relations publiques »; valoriser l'enseignement; réaliser des études commandées et financées.
} 


\section{Etat de la recherche}

Internet $^{198}$. Le CECOM, au fil des années et notamment sous la direction de Pierre de Saint-Georges, a pratiqué une modalité d'intervention et d'expertise de type psychosociologique, attentive aux dynamiques communicatives en tant que dimension constitutive des organisations ${ }^{199}$. En 1994 notons la parution du premier numéro de la revue Recherches en communication, devenue un lieu d'expression majeur des activités de recherche dans le domaine de la communication des organisations à l'UCL ${ }^{200}$.

Le LASCO est né dans sa forme actuelle le 7 février 2000, au sein du département de communication de l'UCL, comme "groupe à dénomination particulière ». Les trois fondateurs en sont Axel Gryspeerdt (directeur du laboratoire jusqu'en 2009), Vincent Defourny et Pierre de Saint-Georges. Au moment de sa fondation, il comptait onze membres effectifs, professeurs et assistants, et une série de membres associés, provenant surtout de la faculté des sciences politiques, économiques et sociales de l'UCL, mais également d'autres institutions (hautes écoles et instituts de formations, par exemple), et en particulier de l'IHECS (Institut des Hautes Etudes des Communications Sociales à Bruxelles).

Le site du laboratoire rappelle que le LASCO regroupe des chercheurs "venus d'horizons différents, mais fédérés autour de l'observation et de l'analyse des phénomènes de communication interne et externe, stratégique ou spontanée des organisations ; ces phénomènes étant considérés comme la conséquence et l'origine des interactions développées au sein ou à partir des organisations ». Au centre de l'attention des fondateurs du LASCO était donc la connexion entre communication et organisation, entendue comme phénomène crucial pour notre temps, à étudier à partir de différents points de vue disciplinaires. Cette attention centrale a guidé les travaux du laboratoire, articulés selon différents axes présentés dans les documents de fondation du laboratoire (voir Gryspeerdt 2000, site du LASCO). En premier lieu, nous trouvons l'analyse de la

\footnotetext{
${ }^{198} \mathrm{http} / / / \mathrm{cecom} . c 0 m u . u c l . a c . b e / p r e s e n t a t i o n . h t m$, consulté le 15 septembre 2009.

${ }^{199}$ Voir, outre le site du CECOM, Agnès d'Arripe 2009.

${ }^{200}$ Parmi les « ancêtres » du LASCO, Agnès d'Arripe $(2009,4)$ rappelle les travaux de Vincent Hanssens et de Didier van den Hove, qui ont poursuivi au sein de l'UCL une approche psychosociologique de la communication, des organisations et du management dans une perspective proche de l'Association pour la Recherche et l'Intervention Psychosociologiques (ARIP) de Paris (aujourd'hui CIRFIP).
} 
communication interne, entendue comme «l'ensemble des comportements en interaction des différents acteurs de l'organisation » dans un contexte spécifique. En deuxième place, se trouve l'analyse des stratégies de communication interne et externe, et donc «l'étude de l'action délibérée d'acteurs professionnels cherchant à influencer ou à faire évoluer la configuration des rapports sociaux, internes et/ou externes, constitutifs des organisations aussi bien dans les secteurs marchands et non-marchands que publics». Enfin, l'attention se porte sur les cadres théoriques et conceptuels, présents dans la pratique et dans la réflexion sur la communication d'organisation, autrement dit, le nécessaire volet méthodologique et épistémologique de la recherche. Les exemples de thématiques concrètes à affronter sont bien connus : l'analyse de la communication dans les organisations comme lieu de production de culture (en particulier, à travers les rites); la légitimation; la régulation; le changement organisationnel et les autres dynamiques des organisations en relation avec la communication; la médiation; les métiers et pratiques professionnelles.

Comme le souligne Agnès D'Arripe (2009, 7), les activités du LASCO pendant les dix ans qui nous séparent de sa fondation montrent bien l'existence de deux directions complémentaires de recherche. Pour reprendre le couple conceptuel introduit par Pierre de Saint-Georges (2006), il s'agit de l'attention pour la « communication organisationnelle », l'ensemble de comportements de communication qui sont constitutifs de toute organisation, et de l'étude de la « communication d'organisation", les actes de communication du sommet de l'organisation et donc liés à la «culture-projet». La première direction trouve ses racines dans l'œuvre de Vincent Hanssens et Didier van den Hove. Elle est représentée par des auteurs comme Pierre de Saint-Georges, Joseph Pirson, Claude Duterme, Laura Salamanca, Florence Carion et autres ; la deuxième, initiée par Vincent Levaux, par les travaux d'Axel Gryspeerdt, Vincent Defourny, Thierry Libaert et Jean-Marie Pierlot.

Les sources disciplinaires des travaux du LASCO indiquées dans le site sont au nombre de trois : la sociologie, la psychosociologie des organisations et de la communication et la médiologie. Plus en détails, en suivant encore Agnès D'Arripe, on observe la centralité des études en sociologie des organisations (Friedberg et Crozier, Sainsaulieu, Mintzberg), de la psycho-sociologie, et d'une série d'auteurs belges (souvent liés à l'UCL et même au LASCO) qui ont participé au développement de la conception « orchestrale » de la communication, avec différentes origines disciplinaires (Jean Lohisse, Annabelle 


\section{Etat de la recherche}

Klein, Jean-Pierre Meunier et aussi bien sûr Yves Winkin). Soulignons entre autres l'orientation anthropologique et microsociologique mises en évidence par les recherches sur les relations publiques d'Axel Gryspeerdt, avec une référence importante à l'analyse socio-anthropologique et communicationnelle du rite (Gryspeerdt 1995), et les recherches de Marie-Elisabeth Volckrick sur la médiation.

Même dans un contexte de différences, avec une variété multidisciplinaire d'approches, on peut observer, comme le fait d'Arripe, une certaine cohérence dans les travaux des membres du LASCO, fédérés par l'attention au développement d'une science de la communication comme interdiscipline systémique et pragmatique.

Cet ensemble varié de recherches a conduit à la production d'un nombre important d'articles et d'ouvrages, et à la préparation de nombreuses thèses ${ }^{201}$. Nous citerons seulement trois numéros de la revue Recherches en communication («Esthétique des organisations », «Espace organisationnel et architecture», «Légitimation et communication » ${ }^{202}$ ). Rappelons, qui plus est, l'organisation de séances d'un séminaire de recherche, qui a permis aux membres de discuter d'un nombre important de thèmes liés à la communication des organisations, et d'organiser des conférences entre plusieurs protagonistes $\mathrm{du}$ monde de la recherche sur la communication des organisations. Le LASCO a été un acteur majeur du groupe de contact du Fonds National de la Recherche Scientifique (FNRS) dédié aux Théories des relations publiques et de la communication, fondé par Yves Winkin et Axel Gryspeerdt en 1990. Il s'agit d'une plate-forme de discussion qui permet d'organiser des rencontres annuelles entre les différentes universités francophones de Belgique et d'inviter des protagonistes (souvent étrangers) de ce champ de recherche ${ }^{203}$. En général, l'activité du LASCO a permis la

\footnotetext{
${ }^{201}$ Nous rappelons seulement les thèses actuellement en cours d'élaboration par des membres du LASCO : le travail de Nicolas Baygert sur « le politicien comme marque »; la thèse d'Agnès d'Arripe sur la culture organisationnelle dans un centre universitaire; l'analyse de la présence du Web 2.0 dans les organisations, menée par Karine Johannes; l'étude de la communication interne en condition de crise de Christine Hambursin. Voir aussi : http://www.uclouvain.be/206494.html.

${ }^{202}$ Respectivement n. 17 et 18, 2002, coordonnés par Axel Gryspeerdt et Philippe Marion, et 25, 2006, coordonné par Elisabeth Volckrick.

${ }^{203}$ Parmi les derniers thèmes traités, nous rappelons seulement ceux des quatre dernières rencontres du groupe de contact : la légitimité et l'idéologie
} 
création d'un réseau international de contacts avec d'autres universités, surtout, pour le moment, dans le monde de la francophonie ${ }^{204}$.

\section{Aujourd'hui et demain : nouveaux défis institutionnels et scientifiques}

2009 marque un changement important dans la vie du LASCO, d'un point de vue interne et par rapport à l'environnement extérieur. En 2009, Axel Gryspeerdt et Pierre de Saint-Georges, deux «pères fondateurs » du laboratoire, partent en éméritat, tout en restant actifs au sein du groupe ${ }^{205}$. En même temps, deux nouveaux académiques entrent en fonction au sein du laboratoire et du département de communication de l'UCL: Thierry Libaert, nouveau directeur du LASCO, et Andrea Catellani, auteur de cette présentation. L'année 2009 voit en outre une phase importante de transformation de l'UCL elle-même. Celle-ci est entrée en effet dans un processus de fusion avec quatre autres entités universitaires belges francophones pour créer une nouvelle et plus grande université en 2010. Dans ce processus, l'organisation de l'enseignement et de la recherche sera plus différenciée qu'aujourd'hui entre facultés (centrées sur l'enseignement) et Instituts (centrés quant à eux sur la recherche). Le LASCO va ainsi s'intégrer à un nouveau Centre de Recherche en Communication, faisant partie lui-même du nouvel Institut Langages et Communication (IL\&C). Ces changements à plusieurs niveaux vont influencer la vie future et les projets du LASCO, que nous chercherons ici à résumer brièvement.

L'intégration dans un centre de recherche et un institut aura, on l'espère, des effets positifs: en particulier, celui d'augmenter l'interaction avec les chercheurs en linguistique, et avec les autres groupes de recherche qui s'occupent de différents phénomènes de

dans les organisations (2002), la relation entre nouvelles régulations économiques et nouvelles communications d'organisation (2004), les rites d'initiation en relation avec la vie des organisations (2006), les médias contributifs et l'organisation apprenante (2008).

${ }^{204}$ Parmi les équipes partenaires, nous rappelons seulement l'EUPRERA, le groupe Org \& Co (SFSIC), le GERICO de Lille III, le CELSA et le GRIPIC (Paris IV, Paris Sorbonne), le GREC/O (Laboratoire MICA - Bordeaux 3), l'Université Laval (Canada).

${ }^{205}$ Deux colloques sont organisés à ce propos : «Contredire l'entreprise. Les relations publiques à l'épreuve des contestations » (23 octobre 2009), pour Axel Gryspeerdt, et «Communication et médias au cœur des conflits du travail » (titre provisoire, mars 2010), pour Pierre de Saint-Georges. 


\section{Etat de la recherche}

production de sens et de communication. Notre situation épistémologique actuelle, que quelqu'un a appelée " hypermoderne » (Ascher 2005), est signée par une forte tendance à l'hybridation disciplinaire, au métissage, et à l'exaltation du réseau comme métaphore désormais abusée d'un idéal du savoir et de son organisation. Il s'agit alors de suivre cette tendance, tout en la contrôlant selon une approche scientifique rigoureuse. Nous voyons clairement, de ce point de vue, la nécessité d'un travail épistémologique et méthodologique, exigence déjà bien présente dans les esprits aux temps de la fondation du CETEDI ${ }^{206}$. Les efforts centraux des chercheurs du LASCO resteront donc le dialogue constructif entre différentes disciplines et postures analytiques, avec une préférence pour les approches qualitatives. Pour ce faire, le laboratoire veut devenir un lieu de travail commun, coordonné, capable de produire une accumulation de savoirs et de créer les conditions pour développer des projets importants. La continuité de l'activité de séminaire et l'augmentation de l'esprit d'équipe seront importants de ce point de vue.

Un travail de "veille théorique » sera aussi nécessaire : par rapport aux sources disciplinaires déjà bien connues au LASCO, il faut en effet continuer l'exploration des propositions qui puissent enrichir l'étude des phénomènes communicationnels. Nous pensons par exemple au développement des études narratives appliquées aux organisations (voir par exemple Grant 2004), à la sociologie de la traduction de Bruno Latour, aux sciences de la gestion et aux possibilités offertes par les dernières évolutions de la sémiotique, d'orientation pragmatique-interprétative et poststructuraliste. Toutes ces contributions peuvent nourrir une modalité de recherche qui considère globalement la communication comme lieu d'exercice et de transformation de la culture (Winkin 2001), tout en gardant une ouverture à différentes postures, et en n'oubliant pas les tentatives de constituer une véritable interdiscipline. Il faudra cultiver une approche de la recherche en communication qui soit en même temps attentive à la réalité des phénomènes, à la vie concrète des organisations, et capable de "prononcer » des théories et des hypothèses explicatives généralisables, contrôlables et falsifiables. La fin présumée des grandes narrations épistémologiques ne doit pas signifier la perte de la

\footnotetext{
${ }^{206}$ La réflexion interdisciplinaire sur la situation de l'analyse de la culture aujourd'hui, et entre autres des phénomènes culturels des organisations, est un des projets centraux de l'auteur de cet article.
} 
tension à la construction théorique, mais peut-être une conscience augmentée de la complexité à explorer.

Parmi les lignes de recherche actuellement choisie par les membres du LASCO figurent trois thèmes qui sont à l'ordre du jour : le concept controversé de réseau d'organisation; la relation bidirectionnelle entre éthique et communication, et donc l'éthique de la communication mais aussi la communication sur l'éthique ; l'impact du web 2.0 sur la communication des organisations. Liée au thème de l'éthique, la problématique de la relation entre communication d'organisation et développement durable, et en général la responsabilité sociétale de l'entreprise, attire particulièrement l'attention de quelques membres du laboratoire (Libaert, Catellani ; voir, dans ce même numéro, notre article).

D'un autre côté, le LASCO veut continuer à développer des liens avec la société environnante pour poursuivre le but d'un service à la société qui soit aussi enrichissant pour la recherche, et pour créer (ou plutôt renforcer) des passerelles avec le monde professionnel. Une initiative qui va dans cette direction est celle d'une newsletter périodique électronique, dédié à l'actualité du laboratoire. De nouveaux partenariats sont envisageables, pour continuer par exemple à sonder la réalité des phénomènes d'échange et de tension à l'intérieur des organisations $^{207}$. Un autre grand défi pour le futur sera celui de cultiver et développer les liens interuniversitaires et internationaux existants, et de créer des nouveaux contacts et collaborations avec d'autres universités, en particulier dans le monde anglo-saxon.

En conclusion, le LASCO veut continuer à se proposer comme protagoniste de la recherche sur le lien complexe et changeant entre communication et organisations, dans un contexte qui montre des tendances contradictoires. En effet, d'un côté, nous voyons un intérêt toujours extrêmement vif et même grandissant pour les problèmes de la communication des organisations (par exemple, en contexte de crise), même si, souvent, cet intérêt se réduit à la demande de recettes et solutions "prêtes à porter ». De l'autre côté, nous observons une certaine tendance, pointée par exemple par Agnès d'Arripe en Belgique, à une dévalorisation de ce type d'approche sur le plan scientifique et académique, pour favoriser plutôt la recherche sur les nouvelles technologies et sur les médias. La nécessité, faite sienne par

${ }^{207}$ Les compétences spécifiques dans ce champ, comme celles de Thierry Libaert sur la communication "sensible", environnementale et de crise, seront cruciales dans ce sens. 
Etat de la recherche

le LASCO, de «parler un langage scientifique » adaptable et pénétrant doit répondre à ce double défi, pour proposer un surplus d'intelligibilité sur notre société et sur ses phénomènes de communication. Le nouveau logo du laboratoire (voir ci-dessous) synthétise cet effort en présentant ensemble 1'@, emblème de l'hypermodernité communicative électronique, et la silhouette d'une main, imprimée sur une paroi de la grotte de Lascaux. La double référence, phonétique et visuelle, à cet exemple archaïque de communication, connectée avec l'ultra-modernité de la communication électronique, veut signifier que la communication, objet de l'activité du LASCO, est un élément éternel, en même temps ancien et toujours nouveau, de la vie de l'humanité.

\section{Bibliographie}

ASCHER, F., La société hypermoderne, Éditions de L'Aube. Coll. essais, 2005.

d'ARRIPE, A., "La communication organisationnelle en Belgique : une mosaïque épistémologique et théorique », communication présentée pendant le colloque "Jeunes chercheurs et recherches récentes - La communication des organisations en débat dans les SIC : Quels objets, terrains et théories? », Rennes, 11 et 12 juin 2009.

DEFOURNY, V., Communication, organisation et décision. Contribution à une modélisation systémique du pilotage de la communication dans les organisations, Faculté des sciences économiques, sociales et politiques, Département de communication, Louvain-la-Neuve, 1991.

DE SAINT-GEORGES, P., «Culture d'entreprise, communication interne et stratégies de changement», in Communication \& Organisation, 4, Bordeaux, 1993, p.70-93.

- «Intervention psychosociologique et changement dans les organisations ", in GARANT, M., et SCIEUR, Ph., Organisations et systèmes de formation, Bruxelles : De Boeck, 2002, p. 169 et sq.

- «Métiers de communication et processus de légitimation: le " communicateur-symptôme " " in Recherches en Communication, 25, Louvain-la-Neuve, 2006, p. 53-64.

DUTERME, C., La communication interne en entreprise ; l'approche de Palo Alto et l'analyse des organisations, Bruxelles: De Boeck, 2002.

GRANT, D., The Sage handbook of organizational discourse, London: Sage, 2004. 
GRYSPEERDT, A., Une industrie de la célébration : des relations publiques à la communication d'organisation ; précédé de Jalons pour une histoire des relations publiques, Bruxelles : EVO, 1995.

- «LASCO - Laboratoire d'analyse de la communication des organisations ", In Communication \& Organisation, 19, Bordeaux, 2001, p. 247-251.

- «Relations Publiques et recherche en communication», in Hermès, 38, Paris, 2004, p.148-154

GRYSPEERDT, A., et KLEIN, A., La galaxie des rumeurs, Bruxelles : EVO, 1995.

LIBAERT, T., Communiquer dans un monde incertain, Editions Village Mondial, 2008.

- La communication externe de l'entreprise, Paris : Dunod, 2008.

- Introduction à la communication, Paris : Dunod, 2009.

LIBAERT, T., et PIERLOT, J. M., La communication des associations, Paris : Dunod, 2009.

LIBAERT, T., et WESTPHALEN, M.-H., Communicator - toute la communication d'entreprise, Paris : Dunod, 2009 ( $5^{\text {ème }}$ éd.).

LITS M., "Cinquante années de recherches en communication» in Recherches en communication, 11, Louvain-la-Neuve, 1999, p. 9-19.

MEUNIER, J-P., PERAYA, D., Introduction aux théories de la communication: Analyse sémio-pragmatique de la communication médiatique, Bruxelles : De Boeck Université, 2004.

PIRSON, J., « De l'analyse stratégique à l'analyse pragmatique : une approche communicationnelle des changements organisationnels dans le secteur culturel », In Recherches sociologiques, 3, Louvain-laNeuve, 1999, p. 131-146.

PUUSTINEN, L., AKDOGAN, I., Mapping Media and Communication Research: Belgium, Communication Research Center, University of Helsinki, 2008.

SALAMANCA AVILA, L., La métacommunication comme indicateur central du dispositif d'intervention, Faculté des sciences économiques, sociales et politiques, Département de communication, Louvain-la-Neuve, 2003.

WINKIN, Y., Anthropologie de la communication, Seuil, Paris, 2001. 